\title{
METALLICITY AND AGE OF THE STELLAR STREAM AROUND THE DISK GALAXY NGC 5907
}

\author{
Seppo Laine ${ }^{1}$, Carl J. Grillmair ${ }^{1}$, Peter CapaK $^{1}$, Richard G. Arendt $^{2}$, Aaron J. Romanowsky ${ }^{3,8}$, \\ David Martínez-Delgado ${ }^{4}$, Matthew L. N. Ashby ${ }^{5}$, James E. Davies $^{6}$, Stephen R. Majewski $^{7}$, Jean P. Brodie $^{8}$, \\ R. Jay GaBany ${ }^{9}$, AND JaCOB A. ARnOld ${ }^{8}$ \\ ${ }^{1}$ Spitzer Science Center-Caltech, MS 314-6, Pasadena, CA 91125, USA; seppo@ipac.caltech.edu \\ ${ }^{2}$ CRESST/UMBC/NASA GSFC, Code 665, Greenbelt, MD 20771, USA \\ ${ }^{3}$ Department of Physics and Astronomy, San José State University, One Washington Square, San Jose, CA 95192, USA \\ ${ }^{4}$ Astronomisches Rechen-Institut, Zentrum für Astronomie der Universität Heidelberg, Mönchhofstr. 12-14, D-69120 Heidelberg, Germany \\ ${ }^{5}$ Harvard-Smithsonian Center for Astrophysics, 60 Garden Street, Cambridge, MA 02138, USA \\ ${ }^{6}$ Space Telescope Science Institute, 3700 San Martin Drive, Baltimore, MD 21218, USA \\ ${ }^{7}$ Department of Astronomy, University of Virginia, P.O. Box 400325, Charlottesville, VA 22904-4325, USA \\ ${ }^{8}$ University of California Observatories and Department of Astronomy and Astrophysics, University of California, \\ 1156 High Street, Santa Cruz, CA 95064, USA \\ ${ }^{9}$ Black Bird Observatory, 5660 Brionne Drive, San Jose, CA 95118, USA \\ Received 2016 February 22; revised 2016 June 15; accepted 2016 June 23; published 2016 September 6
}

\begin{abstract}
Stellar streams have become central to studies of the interaction histories of nearby galaxies. To characterize the most prominent parts of the stellar stream around the well-known nearby $(d=17 \mathrm{Mpc})$ edge-on disk galaxy NGC 5907, we have obtained and analyzed new, deep gri Subaru/Suprime-Cam and $3.6 \mu \mathrm{m}$ Spitzer/Infrared Array Camera observations. Combining the near-infrared $3.6 \mu \mathrm{m}$ data with visible-light images allows us to use a long wavelength baseline to estimate the metallicity and age of the stellar population along an $\sim 60 \mathrm{kpc}$ long segment of the stream. We have fitted the stellar spectral energy distribution with a single-burst stellar population synthesis model and we use it to distinguish between the proposed satellite accretion and minor/major merger formation models of the stellar stream around this galaxy. We conclude that a massive minor merger (stellar mass ratio of at least 1:8) can best account for the metallicity of -0.3 inferred along the brightest parts of the stream.
\end{abstract}

Key words: galaxies: evolution - galaxies: interactions - galaxies: structure

\section{INTRODUCTION}

Major mergers, mergers that take place between galaxies of comparable mass (say stellar or total mass ratios of 1:3-1:5 or larger, with the ratio being the mass of the lower mass galaxy over the mass of the more massive galaxy), have attracted attention in the past (e.g., Arp 1966; Schweizer 1982; Hibbard \& van Gorkon 1996; Bush et al. 2008) because they have features that are easily detected even in shallow imaging observations. However, major mergers may be red herrings when studying the evolution of typical galaxies, many of which may not have undergone a major merger event since $z=1$ (e.g., Xu et al. 2012). Much more common (e.g., RodriguezGomez et al. 2015) are "satellite accretion events" (stellar or total mass ratio of mostly $\lesssim 1: 50$; e.g., Deason et al. 2016) that are mergers of a dwarf galaxy with a massive parent galaxy. Evidence for such cannibalism has been found around our own Milky Way galaxy, including the Sagittarius Dwarf stream (e.g., Ibata et al. 2001b; Majewski et al. 2003), Monoceros Ring (Newberg et al. 2002), Anticenter Stream (Grillmair 2006b), Orphan Stream (Grillmair 2006a; Belokurov et al. 2007), and Styx Stream (Grillmair 2009). Such streams have also been seen around the Andromeda galaxy (e.g., Ibata et al. 2001a).

The effects of mergers intermediate in strength between major mergers and satellite accretion events, a.k.a. "minor mergers" (stellar or total mass ratios of roughly between 1:50 and 1:5), have proven to be much harder to observe directly. Such mergers are not expected to transform galaxies as drastically as major mergers (disk to elliptical transformation), but on the other hand one would expect the effects of such mergers to show up as more than just tenuous stellar population changes in the halo (see local group streams mentioned above). Moreover, Stewart et al. (2009) have argued that every large galaxy has undergone at least one minor merger during its lifetime. Thus, it can be argued that it is the minor mergers that are the most dynamically significant merging events, in view of their relatively high frequency (see also Zaritsky \& Rix 1997; Bullock \& Johnston 2005) and the substantial dynamical and structural impacts on the primary galaxy that such interactions can bring about. These impacts include the heating and thickening of host galaxy disks, growth of galactic bulges, hierarchical build-up of galaxy mass, counterrotating cores in host galaxies, and triggering and maintenance of bar and spiral structures (e.g., Mori \& Rich 2008; Purcell et al. 2010). Observational evidence for minor mergers has been surfacing during the last decade, as fairly high surface brightness stellar streams around several nearby galaxies have been detected in ground-based visible-light observations (e.g., Shang et al. 1998; Martínez-Delgado et al. 2008, 2009, 2010; Duc et al. 2015; Jennings et al. 2015).

NGC 5907 is a nearby $(d=17 \mathrm{Mpc}$; Tully et al. 2013, $\mathrm{AB}_{\text {mag }}{ }^{3.6}=-21.9 ;$ NASA/IPAC Infrared Science Archive, $V_{\mathrm{rot}}^{\max }=240 \mathrm{~km} \mathrm{~s}^{-1}$; Casertano 1983) edge-on Sc-type disk galaxy with a stellar stream around it, discovered by Shang et al. (1998), and studied further by Zheng et al. (1999) and Martínez-Delgado et al. (2008). The outer disk of NGC 5907 also has a warp (e.g., Shang et al. 1998). Martínez-Delgado et al. (2008) obtained very deep images of NGC 5907 and revealed the spectacular long stellar stream (Figure 1). However, because they used a luminance filter (see Figure 1 

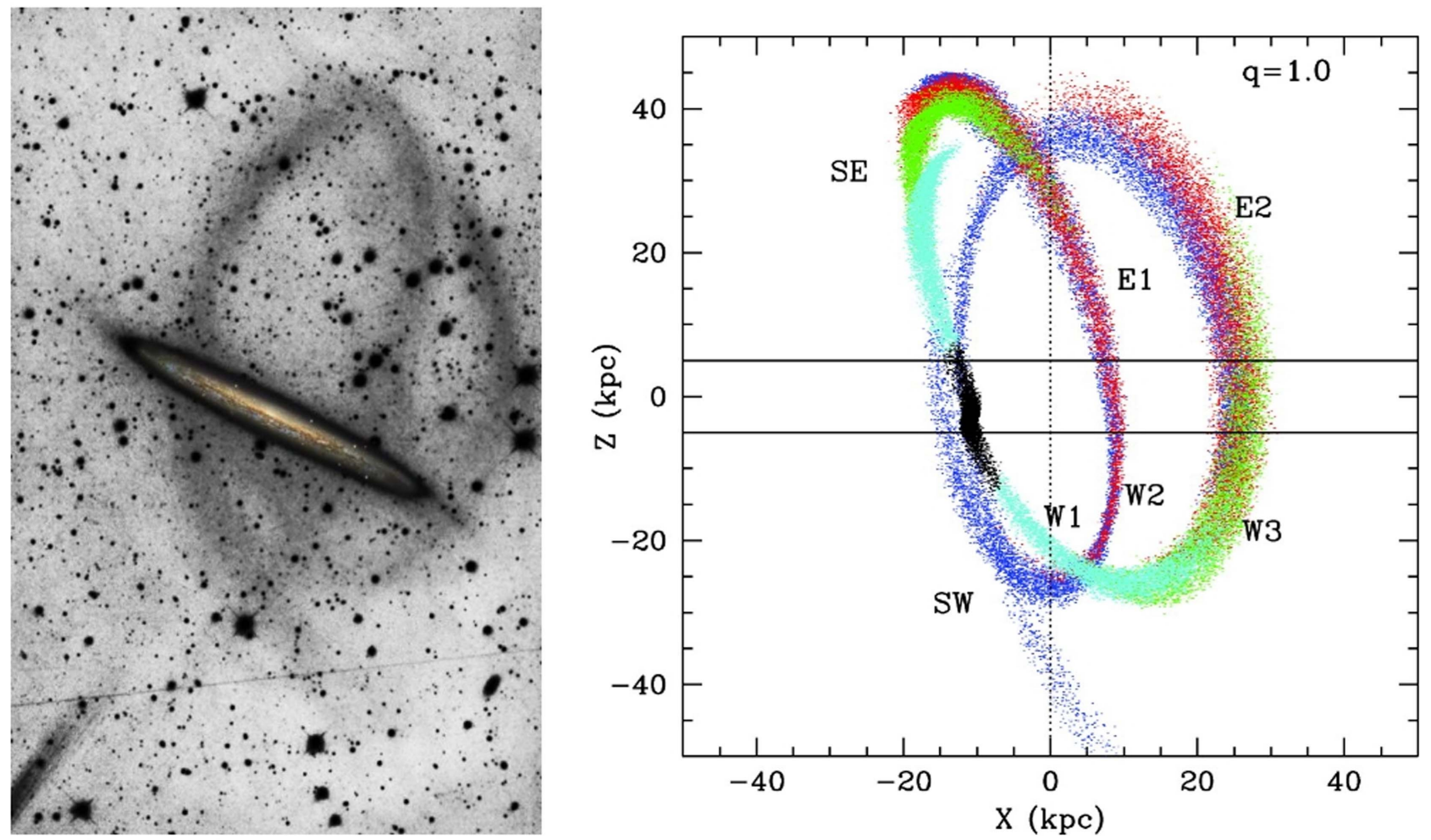

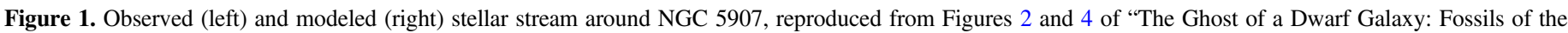

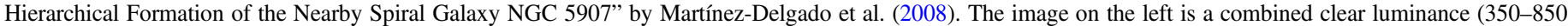

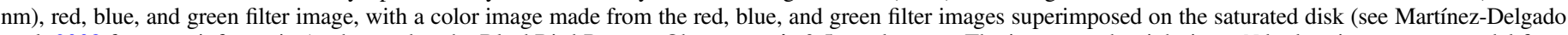

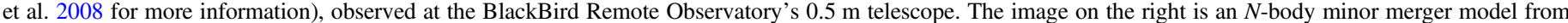

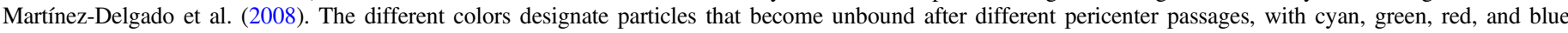

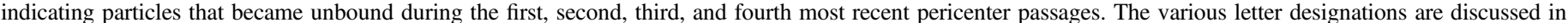

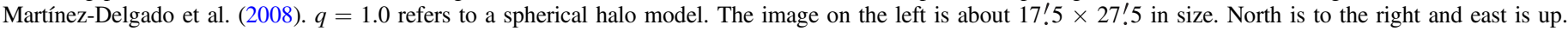

in Martínez-Delgado et al. 2015), Martínez-Delgado et al. (2008) were not able to measure colors in the stream. MartínezDelgado et al. (2008) also produced a simple $N$-body model that mimicked the observed loopy stellar stream as a fossil of the tidal disruption of a single satellite in a merger event (total mass ratio of 1:4000), rejecting the hypothesis of multiple merger events in the halo of NGC 5907.

In contrast, Wang et al. (2012) reported a model that reproduced the general structure of the stellar stream around NGC 5907 in a major merger scenario. Wang et al. (2012) suggested that the colors of the stream may be used to distinguish between a minor satellite accretion event and a major merger origin of the stellar stream in NGC 5907 (the stream is too faint to be observed spectroscopically). They used both (1) the color-inferred iron abundance in the disk outskirts (affected also by stars in the inner halo) and found that the $R-I$ color from Zheng et al. (1999) is similar to that of massive disk galaxies instead of dwarf satellite galaxies, and (2) the mass-metallicity relation in which, for example, the low-mass Sagittarius satellite galaxy (with low $[\mathrm{Fe} / \mathrm{H}]$ ) would produce a bluer color than what is observed. We exploit a similar technique in this paper. We have obtained new deep Subaru/Suprime-Cam gri and Spitzer/Infrared Array Camera (IRAC) $3.6 \mu \mathrm{m}$ images of NGC 5907 to measure the spectral energy distribution (SED) and color indices of the brightest parts of the stellar stream east of the disk of NGC 5907, and to compare them to the predictions of satellite galaxy accretion and minor/major merger models. To our knowledge this is the first time that IRAC data have been used to constrain the metallicity and the age of the stellar population in a stellar stream around a nearby galaxy (outside the local group).

This paper is organized as follows. In Section 2, we summarize the new observations and data. In Section 3, we present the surface brightness and color index results and study the potential effects of an extended point-spread function (PSF). Section 4 addresses how we fit single-burst SED models from the flexible stellar population synthesis (FSPS) code. We discuss our results in Section 5 and present our conclusions in Section 6.

\section{OBSERVATIONS AND DATA REDUCTION}

\subsection{Spitzer/IRAC Observations and Data Reduction}

Spitzer/IRAC (Fazio et al. 2004; Werner et al. 2004) observed NGC 5907 at $3.6 \mu \mathrm{m}$ on 2010 July 12, 14, and 20. We used three separate pointings of IRAC's $5 ! 2 \times 5 ! 2$ field of view (observation identification numbers or AORKEYs 34781952 , 34782208, and 34782464 in program 60088; see Figure 2) that covered the brightest parts of the stream to the east of the galaxy disk. We used 30 positions in a custom offset Reuleaux triangle pattern (Arendt et al. 2000) with a scale of about 75 arcsec and performed a five-point, small-scale (25 arcsec) cycling dither pattern around each point in the triangle for each of the three pointings. The frame time used was $100 \mathrm{~s}$ (which gives $93.6 \mathrm{~s}$ of exposure time per frame at 


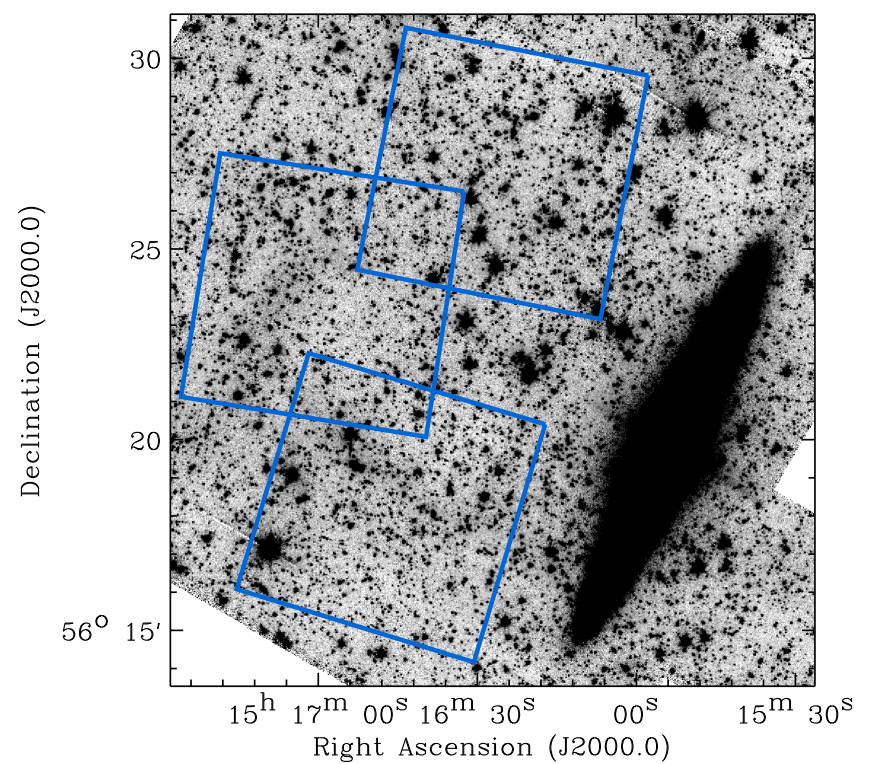

Figure 2. Overlay of the mean location of all the IRAC frames in our new observations on top of an IRAC $3.6 \mu \mathrm{m}$ image from earlier NGC 5907 observations in Spitzer program 3. The boxes drawn have been expanded to show the full area covered by all dithered frames of the three new deeper pointings.

$3.6 \mu \mathrm{m})$. We consequently spent $14,040 \mathrm{~s}$ per field on the stream. Previous data from Spitzer program 3 with ten $100 \mathrm{~s}$ frames had hinted at the brightest parts of the stellar stream (see Figure 2). Our new observations are a factor of 15 longer in exposure time.

Production of the final mosaiced images made use of several tools. The processing started with the corrected basic calibrated data (CBCD) frames produced by version S18.18.0 of the IRAC pipeline. The data for each astronomical observing request (AOR; corresponding to a single dithered field of view) were then self-calibrated using the procedures developed by Fixen et al. (2000) to derive delta-corrections to the detector offsets and to fit time-dependent drifts in the sky intensity (whether due to instrumental effects or changes in the zodiacal light). We then ran the data through a custom column pulldown artifact corrector written by one of us (M. Ashby). The data were again self-calibrated using all three AORs together to derive delta-corrections to the offsets (essentially zero at this point) and the best fit to time-variable offsets. These variable offsets optimize the background matching where there is partial overlap between the three fields.

Subsequently, to obtain the best astrometric solution, we ran the data through one iteration of the Great Observatories Origins Deep Survey (GOODS) pipeline (Grumm et al. 2005) artifact mitigation. Astrometry in the processed CBCD frames was refined using the standard Spitzer Science Center (SSC) provided distortion solution. Rotation and translation parameters for all individual CBCD frames were calculated after distortion had been removed. The rotation and translations were based on both internal references (same source in multiple CBCD frames) and external references in the form of Sloan Digital Sky Survey (SDSS) sources covering the whole NGC 5907 field. More weight was given to the internal match so that our PSFs are very sharp. The result is that the astrometry in our frames is tied to SDSS astrometry.
We then used the GOODS pipeline mosaicer to mosaic the modified CBCDs. Remaining cosmic-ray hits were removed using outlier rejection when the CBCD frames were combined into a mosaic. Some differences of the GOODS pipeline mosaicing compared to the standard SSC pipeline mosaicer include the following.

1. Less astrometric uncertainty (because of the very sharp PSFs) in the mosaics coming from the GOODS pipeline. We weighted internal matches (particular sources seen on many CBCD frames) as indicated above. This was important when we removed sources by PSF fitting below.

2. The drizzle algorithm used to reconstruct an image from undersampled pointings gives superior resolution compared to Spitzer custom software mosaicer and point source extractor (MOPEX) mosaics built with the default MOPEX mosaicing method.

Because stars at the distance of NGC 5907 are completely unresolved in our images, we removed all detected point sources. We ran the SSC-provided custom software astronomical point source extractor for MOPEX (APEX) to find point sources and removed them using the APEX quality assurance pipeline. We opted to remove sources that were centrally concentrated (aperture flux density ratio within a 10 pixel to a 3 pixel radius was less than 1.3 ) or very faint sources that turned out to be essentially point-like. Once we had removed the point sources via PSF fitting, we still had the extended sources left. We used SExtractor (Bertin \& Arnouts 1996) to mask the extended sources. We extended the masks by two pixels in radius in Adobe Photoshop to remove a visible halo around the extended sources. Because the IRAC $3.6 \mu \mathrm{m}$ PSF is fairly steep within the core (it drops by a factor of about 500 within a 5 arcsec radius), the outlying parts of the PSF are well within the noise in the mosaic image for all but the brightest sources (see also Section 3.2 below). Typical slightly extended background sources in the IRAC images have a central pixel value of about $0.05 \mathrm{MJy} \mathrm{sr}^{-1}$ while the $1 \sigma$ noise in the final mosaic is about $9 \times 10^{-4} \mathrm{MJy} \mathrm{sr}^{-1}$, and the brightness of these sources at $3.6 \mu \mathrm{m}$ drops below the noise level within about two native pixels or about 2 ! 5. The amount of light from extended PSFs due to the bright edge-on galaxy disk and the brightest stars in the field of view is further discussed in Section 3.2 below.

\subsection{Subaru Observations and Data Reduction}

NGC 5907 was observed using the Suprime-Cam imager (Miyazaki et al. 2002) consisting of ten $2048 \times 4096$ pixel charge-coupled devices (CCDs) with a total field of view of $34^{\prime} \times 27^{\prime}$ covered by 0 "' 20 pixels, mounted at the prime focus of the $8.2 \mathrm{~m}$ Subaru telescope, on the morning of 2010 April 12. We obtained images through the SDSS gri filters in photometric conditions with 0 ". $6-0$ ". 9 seeing. Between four and five exposures were obtained per filter to help with cosmicray rejection and to increase the dynamic range of the final coadded mosaics. Additionally, the telescope pointing was dithered slightly between exposures to account for bad pixels/columns and to fill the gaps between individual CCDs.

The fully depleted charge-coupled device detectors installed on Suprime-Cam in 2009 January are each divided into four regions read out by separate sets of electronics with different bias levels and gains. We did not use the standard pipeline 
reduction for the Subaru Suprime-Cam data because it is important for us to remove all the sources of underlying background emission (whether instrumental or from the sky) as carefully as possible. In our reduction the bias was first subtracted using the overscan area of the images as defined in the headers and the gain was scaled to 1.0 (everything converted to electrons) for all regions on all chips. Then, a median bias image was created from a set of bias frames and subtracted to remove residual structure from the edges of the readout regions. Next, a median dome flat was constructed for each band, and divided out of the data frames. This left images that were largely flat in the central region of the camera, but with significant large-scale structure in the sky due to scattered light in the frames, and to a lesser degree in the dome flats (Capak et al. 2007).

To remove the sky background, a median sky image was created from the data frames in each band. To avoid oversubtracting extended structure, a two-step masking process was used. First, object detection was run on each frame and used to make a preliminary mask that, in turn, was used to make a first pass sky image. This was then scaled to the background of each exposure and subtracted from the images. This first pass skysubtracted image was then combined into a mosaic, and a manual mask was made of all extended emission regions visible in the mosaic, ensuring that we are not accidentally removing parts of the stream. The manual mask was then propagated back to the individual images, and a second pass sky frame made. This second pass sky frame was then inspected, noisy regions masked, and fitted with a tessellated surface. The tessellated surface was then scaled to the background in each exposure and subtracted.

Finally, to remove scattered light that varies from exposure to exposure, each sky-subtracted exposure was masked with both the detected object mask and the extended source mask, divided into an $8 \times 16$ grid, and fitted by a third-order twodimensional polynomial over that grid, which was then subtracted. After attempting fits with higher and lower order polynomials, we settled on the third-order polynomial. That fit to the grid successfully removed the scattered light (which varies only on large scales, $\sim 7^{\prime} \times 14^{\prime}$ ) in the center of the frames, but did not subtract the extended emission. After this process, some residual scattered light was visible around the edge of the frame where the scattered light had more structure (Capak et al. 2007).

We used the following color-corrected magnitude zero points to convert the counts into magnitudes: $g_{\text {zp }}=35.95$, $r_{\mathrm{zp}}=36.32$, and $i_{\mathrm{zp}}=36.19$ for individual frame exposure times of 203, 119, and $142 \mathrm{~s}$ in $g, r$, and $i$, respectively. These zero points are good to 0.002 mag random error for $g$ and $r$ and to $0.003 \mathrm{mag}$ random error for $i$ relative to SDSS, using the color conversions in Capak et al. (2007) that are valid for colors $-1<g-r<1$ for $g$ and $r$ and $-1<r-i<1$ for $i$. The zero point calibration was done against SDSS Data Release 8 with SExtractor source extractor auto magnitudes, using 453, 455 , and 525 stars in $g, r$, and $i$, respectively.

We masked out extended objects by hand in the stream in the $g, r$, and $i$ images. We then cross-convolved the Subaru images with the IRAC PSF and the IRAC $3.6 \mu \mathrm{m}$ image with the Subaru PSFs. The Suprime-Cam gri PSFs were generated by SSC's pfr_estimate.nl Perl script. We subsequently applied a kernel to taper the PSFs to avoid ringing in the cross-convolved

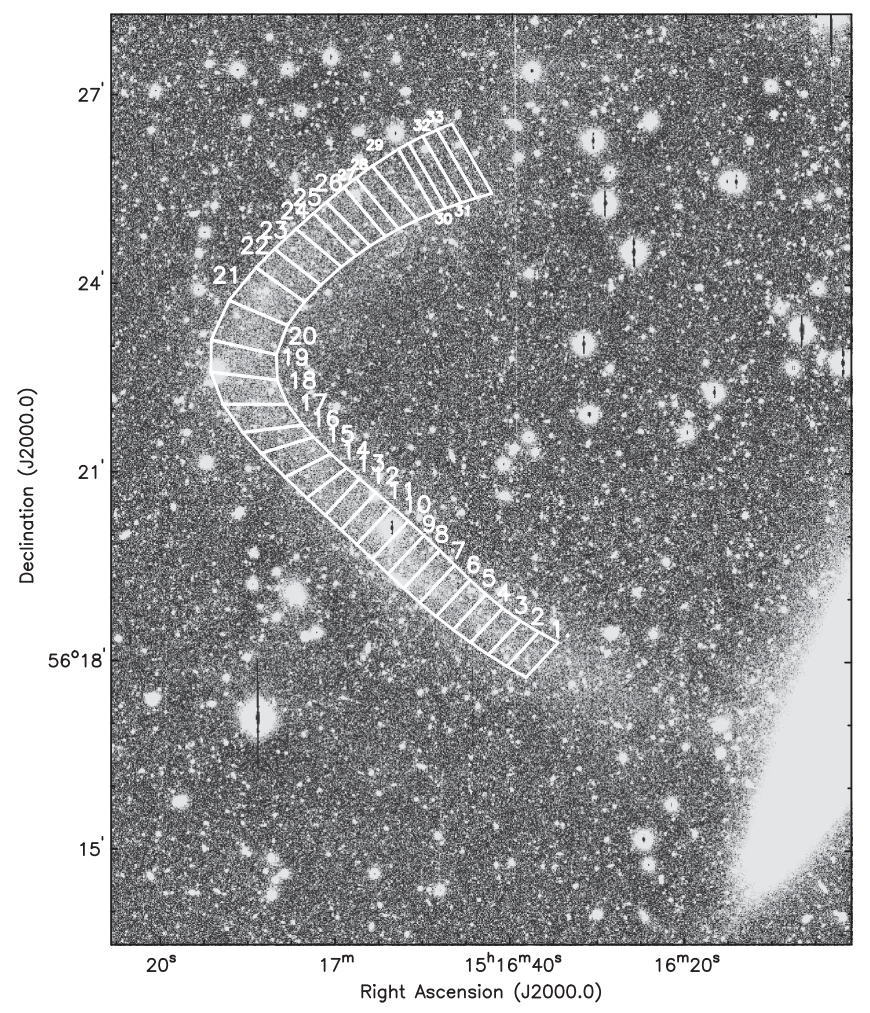

Figure 3. Photometry apertures on top of a Subaru Suprime-Cam $r$-band image. The apertures in which the surface brightnesses and colors were measured are shown on top of an $r$-band Subaru Suprime-Cam image of the stellar stream in NGC 5907. The apertures correspond to roughly 4-5 kpc $\times$ $2-3 \mathrm{kpc}$ each. Even though in this $r$-band image the stream continues to the southwest (SW) of aperture 1, the surface brightness was so low there in the other bands that no attempt was made to measure the stream surface brightness SW of aperture 1 .

images. Color index images were then made by combining the mosaiced images from the various observed bands.

\section{SURFACE BRIGHTNESSES AND COLOR INDICES}

\subsection{Estimation of Colors and Surface Brightnesses}

We analyzed the color index images by constructing apertures, shown in Figure 3, of the stream, and measured the median color of the color index images (Figure 4). The resulting color plots (Figure 5) show the median colors versus the number of the aperture as shown in Figure 3. Mean colors and several other properties of the stream are also shown in Table 1. We tested the robustness of the colors by making Subaru Suprime-Cam images that used the first pass skysubtracted image (see Section 2.2) in the background subtraction (colors changed by less than $0.05 \mathrm{mag}$ ) and by increasing the masked areas within the stream by $5-10$ pixels on each side (colors changed by less than $0.1 \mathrm{mag}$ ). Finally, by measuring the variance in "empty" sky regions outside the stream, we estimated the uncertainty in the sky background value subtraction in the IRAC image to be about 0.08 mag. Therefore, we conservatively believe that our measured colors are accurate to $0.2 \mathrm{mag}$. Extinction corrections due to the Milky Way are expected to be less than 0.02 mag in the visual colors and even less for the visual-infrared colors, and therefore have not been applied, as their effect is much smaller than the other uncertainties in the colors.

In general, the surface brightness is highest in apertures 5-10 and decreases in both directions outside those apertures. 

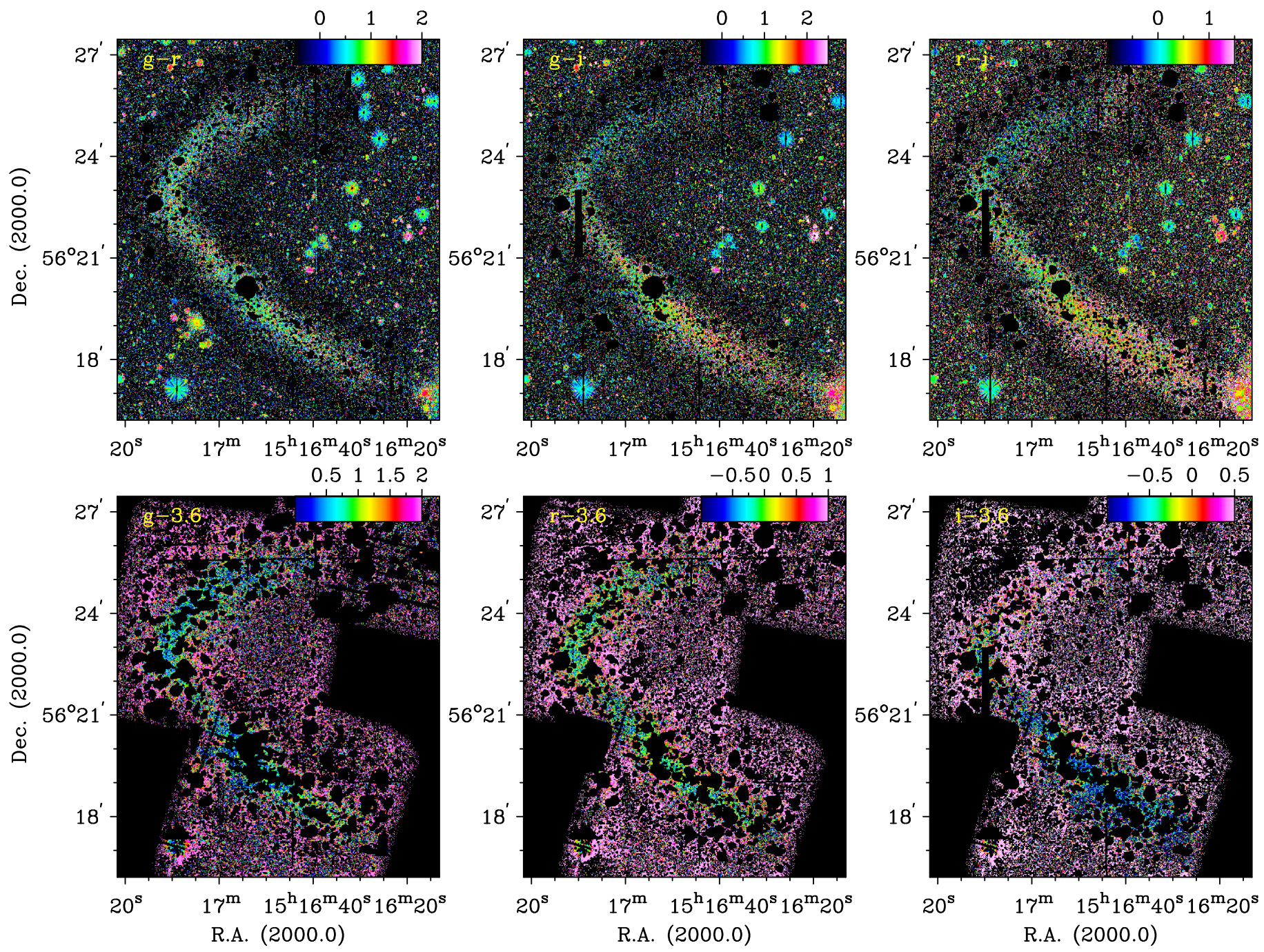

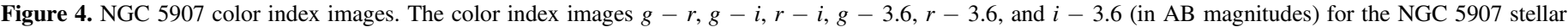

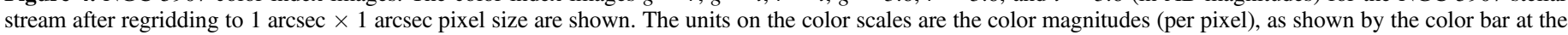

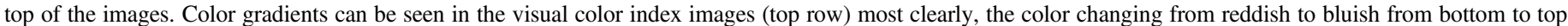
along the stream. See the discussion of these gradients in Section 3.1.

Specifically, the surface brightnesses peak at about 27.4, 26.7, 26.1 , and $26.7 \mathrm{AB}$ mag $\operatorname{arcsec}^{-2}$ in $g, r, i$, and $3.6 \mu \mathrm{m}$, respectively, and are at their faintest toward the northern end of the detected area at 28.5, 28.0, 27.8, and 27.6 AB mag arcsec ${ }^{-2}$ in $g, r, i$, and $3.6 \mu \mathrm{m}$, respectively. The brightest parts of the stream have a transformed $R-I$ color of about 0.7 using the transformation equations of Jester et al. (2005) $(R-I \approx r-i$ $+0.2)$, which is in the range of earlier estimates of $R-I=0.5$ (Zheng et al. 1999) to 0.9 (Shang et al. 1998) in the brightest part of the stream (though note that they measured a different location beyond the sharp turn toward northwest in the stream). In general, there is perhaps a slight gradient in the visual colors toward blue as the aperture number increases, as seen in Figure 5. For example, in $g-i$ the average of the median colors in apertures $1-15$ is 1.3 and the average in apertures $16-23$ is 0.9 . While this gradient is within the uncertainties of our colors, it could be consistent with different progenitor star populations that have been stripped at various times as shown in the models of Martínez-Delgado et al. (2008). See also Figure 1 in the current paper where the right side shows with various colors stars that were stripped from the simulated companion at various times. In the IRAC colors this trend is reversed and the colors become slightly redder toward larger aperture numbers along the stream. However, the largest gradients are seen in colors that include the Subaru $i$ band that has the lowest surface brightness of any of the observed bands in the stream, especially in apertures beyond aperture 23 that are therefore not plotted in Figure 5. See Section 3.2 below for a discussion about a possible contribution to the color gradients from the extended PSFs. In general, the colors are roughly constant along the stream.

\subsection{Possible Effects of the Extended PSF}

Formally, the very extended wings of the PSF could spread a small fraction of the light from NGC 5907 into a very faint and large-scale background gradient, dropping away from the galaxy. Such a gradient, if present, could potentially affect the brightness and colors derived for low surface brightness features such as the stellar stream studied here. To evaluate the effect of the extended PSF on our measurements, we have derived and adopted extended PSFs to construct models of the expected background gradients. 


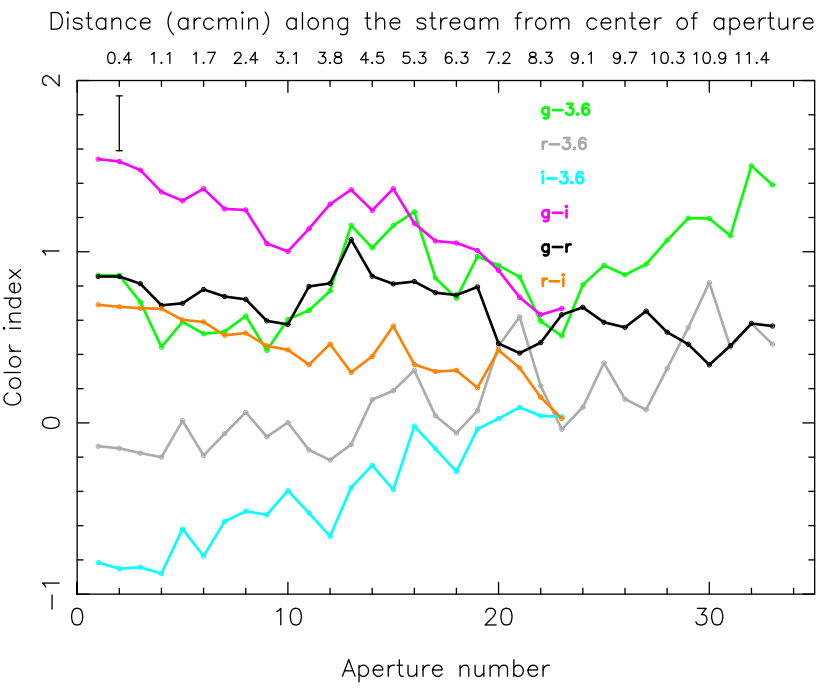

Figure 5. Colors along the stellar stream in NGC 5907. This image shows the AB magnitude colors measured along the stellar stream around NGC 5907 in the apertures shown in Figure 3. The lowest aperture numbers are closest to the galaxy disk, as can be seen in Figure 3. A representative total error bar is drawn in the upper left corner. The uncertainties are much larger for apertures beyond aperture 23 where the stream becomes much fainter. The statistical uncertainties were estimated by dividing the rms in the individual apertures by the square root of the number of independent beams in them.

Table 1

Estimated Properties of the Imaged Stream Section

\begin{tabular}{lccc}
\hline \hline Property & Bandpass & Value & Unit \\
\hline Integrated absolute magnitude & $g$ & -14.1 & $\mathrm{mag}$ \\
Integrated absolute magnitude & $r$ & -14.8 & $\mathrm{mag}$ \\
Integrated absolute magnitude & $i$ & -15.2 & $\mathrm{mag}$ \\
Integrated absolute magnitude & 3.6 & -14.6 & $\mathrm{mag}$ \\
Luminosity & $g$ & $4.9 \times 10^{7}$ & $L_{\odot}$ \\
Luminosity & $r$ & $6.0 \times 10^{7}$ & $L_{\odot}$ \\
Luminosity & $i$ & $7.8 \times 10^{7}$ & $L_{\odot}$ \\
Luminosity & 3.6 & $1.7 \times 10^{8}$ & $L_{\odot}$ \\
Mean $g-r$ & $g, r$ & 0.7 & $\mathrm{mag}$ \\
Mean $g-i$ & $g, i$ & 1.0 & $\mathrm{mag}$ \\
Mean $r-i$ & $r, i$ & 0.3 & $\mathrm{mag}$ \\
Mean $g-3.6$ & $g, 3.6$ & 0.9 & $\mathrm{mag}$ \\
Mean $r-3.6$ & $r, 3.6$ & 0.1 & $\mathrm{mag}$ \\
Mean $i-3.6$ & $i, 3.6$ & -0.1 & $\mathrm{mag}$ \\
Stellar mass & $r$ & $2.1 \times 10^{8}$ & $M_{\odot}$ \\
Mean projected distance to the & $r$ & $9.4 / 46$ & $\mathrm{arcmin} / \mathrm{kpc}$ \\
$\quad$ center of NGC 5907 & & & \\
Detected length of stream & $r$ & $11.7 / 57.8$ & $\mathrm{arcmin} / \mathrm{kpc}$ \\
\hline
\end{tabular}

Note. All the estimates are derived from the apertures shown in Figure 3. All the magnitudes and colors are in the $\mathrm{AB}$ system. Values are measured for apertures $1-33$. The absolute magnitudes are calculated by integrating the flux densities in all the measured apertures and summing them up. The luminosities were calculated using absolute magnitude values of the Sun in the AB system as tabulated in FSPS $(5.12,4.64,4.53$, and 5.99 for $g, r, i$, and $3.6 \mu \mathrm{m}$, respectively). The mass was calculated from the $r$-band luminosity and the $M / L$ ratio (3.46, assuming a Kroupa 2001 type initial mass function, IMF) of the best fitting model in Section 4 . The mean projected distance was calculated as the mean of the distances of the centers of each aperture box 1-33. The detected length is from the center of aperture 1 through the centers of apertures 2-33 and the physical value in kiloparsecs $(\mathrm{kpc})$ is assuming a distance of $17 \mathrm{Mpc}$.

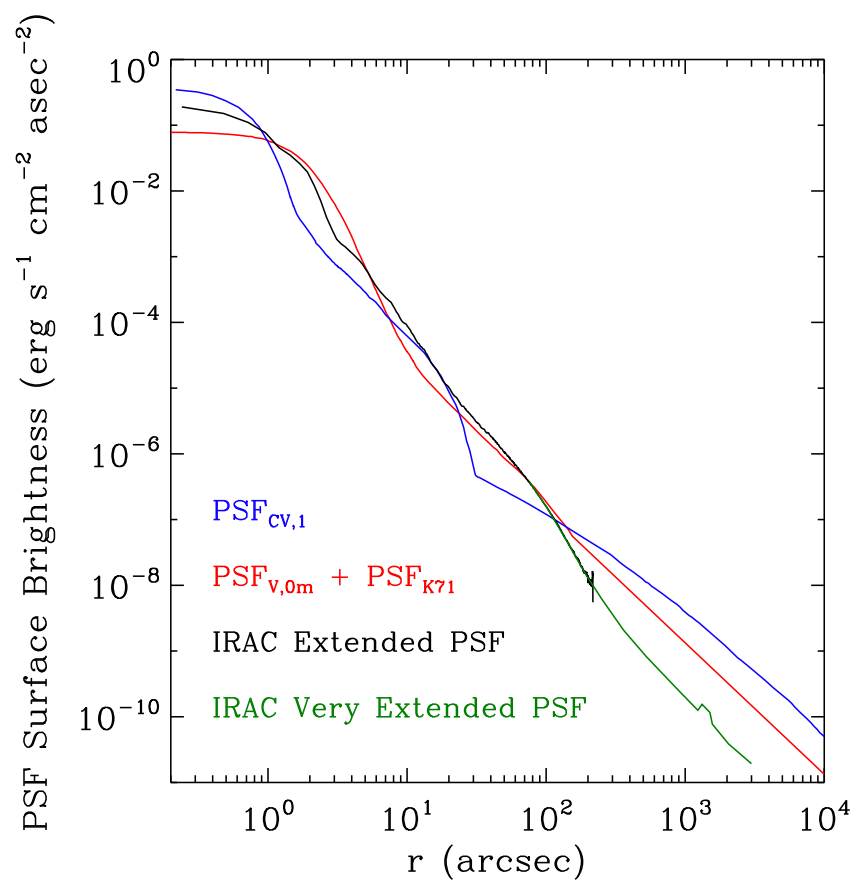

Figure 6. Radial profiles of all the PSFs that were used to model the extended PSF background of NGC 5907. The black and green lines show the IRAC PSF derived from the warm extended PSF and from $\beta$ Gru observations respectively. The red and blue profiles are the two adopted profiles (Sandin 2014) used with the Subaru data.

To derive the radial profile of the extended halo light of the IRAC PSF, we started by constructing the azimuthally averaged profile of the warm IRAC extended PSF. ${ }^{1}$ It provides a radial profile extending to $\sim 200^{\prime \prime}$. The profile at radii from $75^{\prime \prime}$ to $3000^{\prime \prime}$ was determined from serendipitous observations near $o$ Cet (Mira, program id 181), and observations around $\beta$ Gru (program id 1153) that were specifically designed to elucidate the extended IRAC PSF. This very extended profile was normalized to match the radial profile of the abovementioned extended PSF at four points between $63^{\prime \prime}$ and $160^{\prime \prime}$. The agreement in the region of overlap is excellent (standard deviation less than $\sim 4 \%$ ), although the normalization factor required means that the faint ring in the profile at $\sim 1422^{\prime \prime}$ is about 1.7 times brighter than stated in the IRAC Instrument Handbook $^{2}$ (see Section 7.3.4). By combining the two extended PSFs, the dynamic range of the IRAC $3.6 \mu \mathrm{m}$ PSF was improved from about $2 \times 10^{7}$ to about $10^{10}$. The full IRAC PSF to $3000^{\prime \prime}$ is shown in Figure 6.

An analysis of the bright stars in our Subaru images only yielded PSF profiles out to $\sim 40^{\prime \prime}$, and did not show good consistency between cores $\left(0^{\prime \prime}-10^{\prime \prime}\right)$, measured from unsaturated sources, and wings $\left(\sim 5^{\prime \prime}-40^{\prime \prime}\right)$, measured from much brighter, saturated sources. A similar analysis of the bright stars in the COSMOS field (Capak et al. 2007) yielded a better consistency, but was still limited to radial extents of $\lesssim 60^{\prime \prime}$. Therefore, for the Subaru data, we adopted extended PSF profiles, generated from observations made with other telescopes, from the review by Sandin (2014) and references therein. We tested two profiles, to check the sensitivity to the

1 http://irsa.ipac.caltech.edu/data/SPITZER/docs/irac/calibrationfiles/ psfprf/

2 http://irsa.ipac.caltech.edu/data/SPITZER/docs/irac/ iracinstrumenthandbook/ 


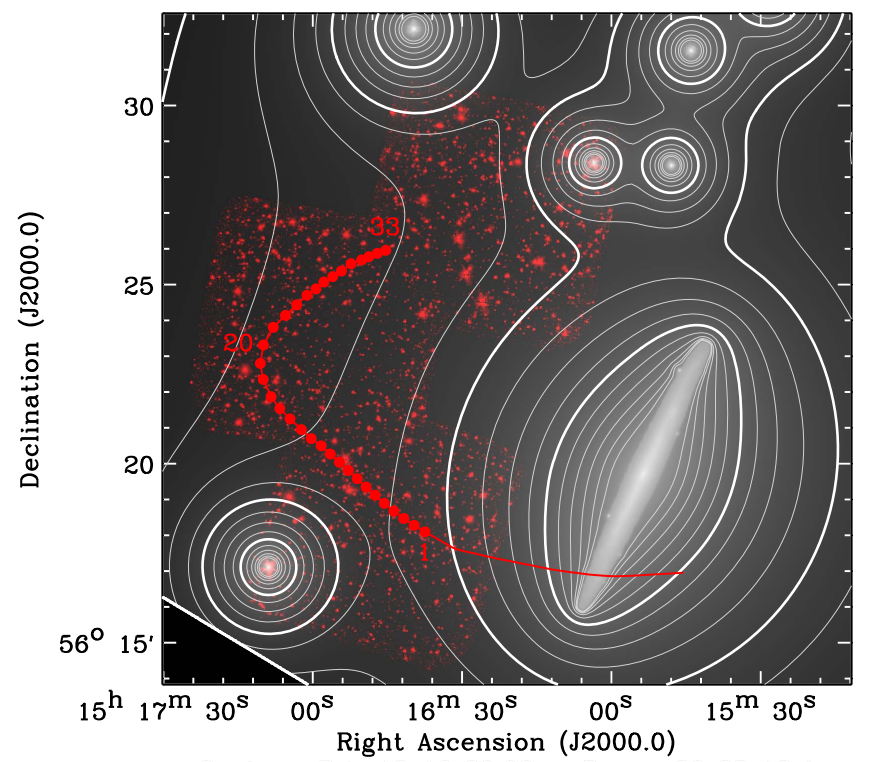

Figure 7. IRAC $3.6 \mu \mathrm{m}$ extended PSF background model. Contours are logarithmically spaced with thick contours at $10^{-5}, 10^{-4}$, and $10^{-3} \mathrm{MJy} \mathrm{sr}^{-1}$. The surface brightness of the stream is at least a factor of 10 higher than that of the extended PSF background light. The centers of the apertures along the stream are indicated by bright red dots. The red line indicates the longer slice depicted in Figure 8. The deep IRAC image is shown in red.

adopted profile. The first tested profile was $\mathrm{PSF}_{\mathrm{V}, 0 \mathrm{~m}}$ (Michard 2002), augmented with $\mathrm{PSF}_{\mathrm{K} 71}$ (King 1971) at radii $>150^{\prime \prime}$. We also tested $\mathrm{PSF}_{\mathrm{CV}, 1}$ (Capaccioli \& de Vaucouleurs 1983) as an alternate PSF. The narrower core of this PSF has an FWHM = 1"!3, which is a closer match to our measured Subaru PSFs at FWHM $\approx 0$ ". 6 . More importantly for our models, the surface brightness of this extended PSF is $\sim 3$ times brighter at $200^{\prime \prime}-1000^{\prime \prime}$ than $\mathrm{PSF}_{\mathrm{K} 71}$. These PSFs are also shown in Figure 6.

To model the background caused by the extended PSF in the IRAC mosaic, we worked with a shallower IRAC mosaic (from Spitzer program 3; see Figure 2) that covered both the stream and the galaxy. We masked the entire PID 3 mosaic except for pixels within $55^{\prime \prime}$ of the galaxy disk and within $30^{\prime \prime}-45^{\prime \prime}$ of the six brightest stars in the field. The stars were included to check if the extended PSF from the bright stars might have a significant effect. We then convolved the masked mosaic with a 2D symmetric kernel generated from the very extended PSF profile in Figure 6. The kernel is normalized such that the total power is conserved in the convolved image. This generates the model that is shown in grayscale and contours in Figure 7. The diffuse emission in the regions where the masked image is nonzero represents the modeled extended PSF halo light from the galaxy and from the six brightest stars in the field. In fact, the light from the extended PSF halo of the brightest stars is found to have little effect on the overall extended PSF light predicted in the area of the stream (see Figure 7). For each Subaru band, two similar models were generated using the profiles in Figure 6, and with the same mask as applied for the IRAC model. The resulting Subaru model images are very similar to the IRAC model, except that the light from the extended PSFs of the stars is less prominent (i.e., underestimated) because the stars are saturated in the Subaru data. The galaxy itself is only saturated in the central $4^{\prime \prime} \times 10^{\prime \prime}$ region of the $i$-band image, which was filled by extrapolation from the $r$-band image before calculating the light in the extended PSF. Slices through all the models are shown in Figure 8.

The models indicate that the expected levels of extended PSF halo light are about a factor of 10 (or more) below the background noise level of the images. The addition of the IRAC extended PSF model to the data has a clear impact on the scale height of the galaxy and on the extended PSF emission surrounding the stars, but no apparent effect at the distance where we measure the stellar stream ( $>100^{\prime \prime}$ from the galaxy). For the Subaru data, the $\mathrm{PSF}_{\mathrm{CV}, 1}$ model has a brighter and flatter background at the $200^{\prime \prime}-600^{\prime \prime}$ radii relevant here.

We tested the effects of the extended PSFs on the measured surface brightnesses and colors of the stellar stream by adding them to the original images (we realize that the extended PSF light component is already in the images, but here we are only interested in the amount of change in the surface brightnesses and colors, so adding instead of subtracting is justified just for this test). In the worst case, using the less steeply falling PSF $\left(\mathrm{PSF}_{\mathrm{CV}, 1}\right)$ for Subaru data, the surface brightnesses changed by $0.21 \mathrm{mag}$ (all the values listed here and in the following are the median surface brightnesses in magnitudes per pixel) at the very northwestern end of the visible stream (in apertures beyond 23 where the stream is very faint) in the $i$ band. However, along most of the stream (apertures 3-23) the change was 0.1 mag or less, and even less for the $\mathrm{PSF}_{\mathrm{K} 71}$ and the IRAC extended PSF.

We also checked the changes in broadband colors along the stream after adding the extended PSF contribution. At worst, the visible-IRAC colors changed by about 0.05 mag (became bluer) along most of the stream (apertures 3-23) and the colors changed even less for the visible-visible bands, with no gradient in these changes along the stream. Making the IRAC surface brightness dimmer by 0.05 magnitudes with respect to the Subaru band surface brightnesses does not change our bestfit results for metallicity and age below.

\section{SED FITTING}

We calculated the median surface brightnesses in the apertures defined in Section 3. Because the stream is getting very faint in at least the $i$ band after its easternmost point (and toward the northwest from there), we calculated the average of the median aperture flux densities in each band, taking into account only apertures 1-23.

We constructed models of single-burst stellar populations with varying ages and metallicities using the FSPS code of Conroy et al. (2009) and Conroy \& Gunn (2010). FSPS makes use of both the Padova (Girardi et al. 2000; Marigo \& Girardi 2007; Marigo et al. 2008) and a "bag of stellar tracks and isochrones" (BaSTI; Pietrinferni et al. 2004; Cordier et al. 2007) stellar evolution calculations in the versions available as of 2009 November. We also used FSPS's Python bindings (Foreman-Mackey et al. 2014). The range in metallicity $\left(\log \left[Z / Z_{\odot}\right]\right)$ in the FSPS/Padova models is from -1.98 to +0.2 and in the FSPS/BaSTI models from -1.80 to +0.32 . The age range that we used was from 0.47 to $14.96 \mathrm{Gyr}$ for the FSPS/Padova models and from 1.86 to $14.79 \mathrm{Gyr}$ for the FSPS/BaSTI models. By scaling the models, we achieved the best least-squares fit to the four average flux densities per pixel for an FSPS/Padova model with a stellar population age of $14.96 \mathrm{Gyr}$ and a metallicity of -0.3 , with a $95 \%$ confidence limit on the metallicity of $-0.3 \pm 0.2$. The best fit is shown in Figure 9, together with an FSPS/Padova model having an age 

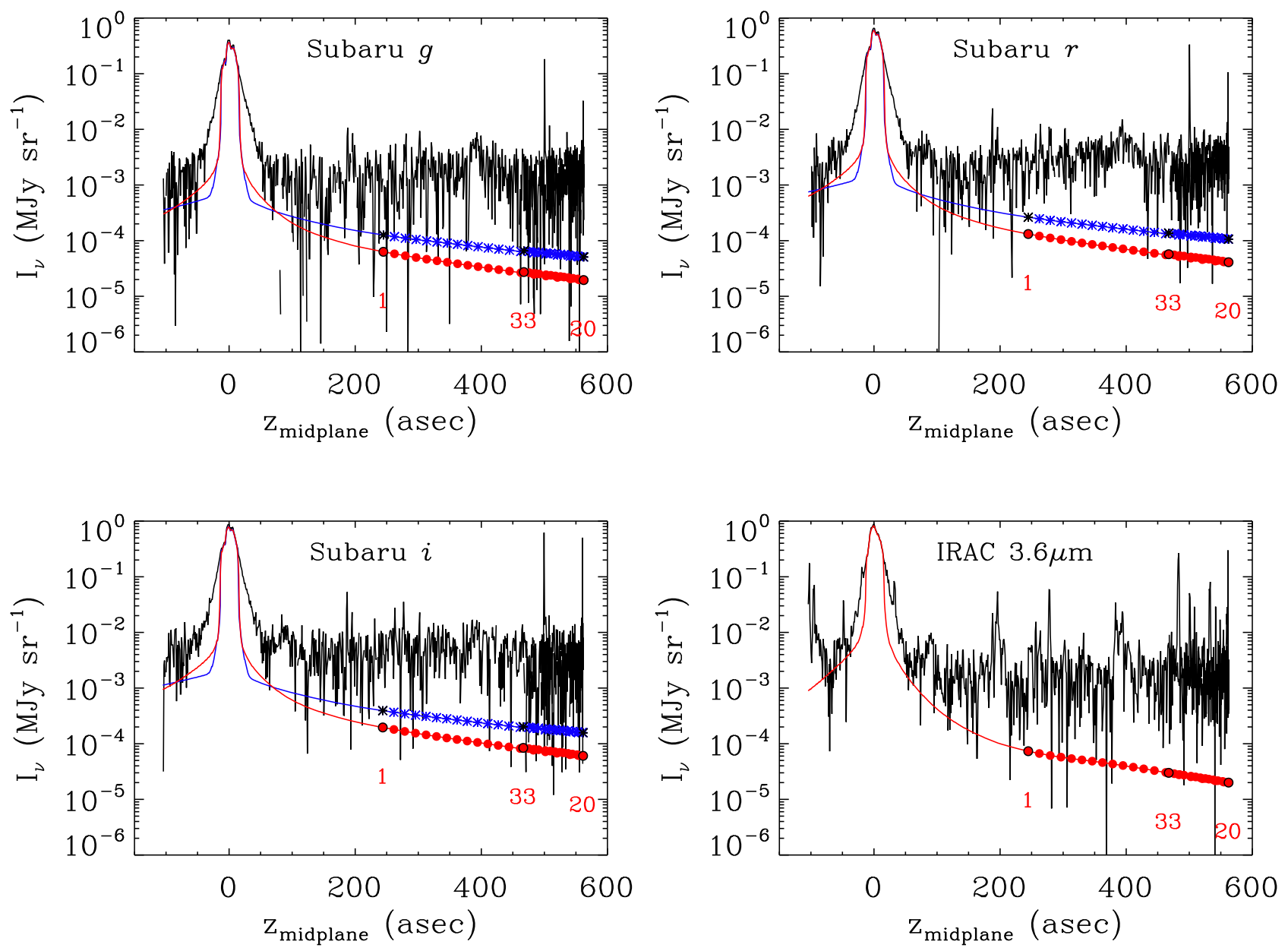

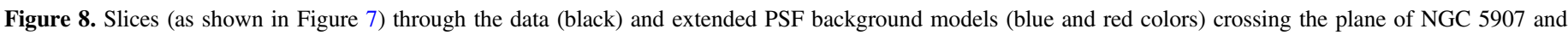

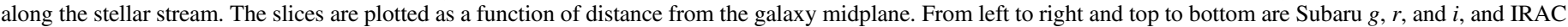

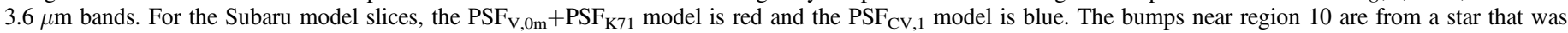
masked out before the measurements were made. Red numerals indicate aperture identifiers as shown in Figure 3.

of $9.44 \mathrm{Gyr}$ and a low metallicity $\log \left[Z / Z_{\odot}\right]$ of -1.98 (showing that old age/low metallicity stellar populations are ruled out). The corresponding $\chi_{2}^{2}$ values of the best fit are shown in Figure 10. The lowest $\chi^{2}$ value for the FSPS/BaSTI models gives a stellar population age around $12 \mathrm{Gyr}$ and a metallicity near -0.3 . It should be noted that including the IRAC $3.6 \mu \mathrm{m}$ band is crucial for the metallicity determination. Averaging the first 12 apertures, the best fitting model has a metallicity of -0.5 and again an age of $14.96 \mathrm{Gyr}$, and averaging apertures 13-23 gives a best metallicity fit of +0.2 and age of $3.6 \mathrm{Gyr}$. These trends in age and metallicity may cause the colors to remain roughly constant. It is possible that despite vigorous masking, there is leftover light from the foreground stars that causes the young metal-rich stellar population to show up in apertures 13-23 when averaged together, as can be seen in Figure 3 (where there were bright foreground stars in the apertures before masking). Excluding the possibly problematic $i$ band (see Section 3.1) from the SED fitting does not change the best fitting stellar population parameters appreciably (best fitting age $11.9 \mathrm{Gyr}$, best fitting metallicity -0.2 ).

The age of the best fitting model may seem problematic because the current best estimate of the age of the universe is about 13.8 Gyr (Planck Collaboration et al. 2015). Basically, the fit is telling us that the oldest possible stellar population provides the best fit. A fit with an age of $10 \mathrm{Gyr}$ is shown with diamonds in Figure 9 and is only slightly worse than the fit with the unphysically large age. The stellar populations in the Sagittarius stream (Chou et al. 2007) and M31's giant stream also have old ages (older than about 7 Gyr; Brown et al. 2006; Tanaka et al. 2010; de Boer et al. 2015).

There are well-known challenges with modeling stellar populations in the infrared. For example, the flux contributions from thermally pulsing asymptotic giant branch stars at $3.6 \mu \mathrm{m}$ are uncertain (see Villaume et al. 2015), and if high, they could shift our solution to a somewhat younger age and lower metallicity. In addition, there is a general tendency for stellar population synthesis models, including FSPS, to predict overly blue visible-light to near-infrared colors (e.g., Conroy \& Gunn 2010), which means that our metallicity inference could be too high. On the other hand, our inference was driven not only by the near-infrared, but also by the relatively red visible-light colors of the stream.

\section{DISCUSSION}

The stellar stream in NGC 5907 has been previously modeled with a satellite galaxy accretion event (MartínezDelgado et al. 2008, total mass ratio 1:4000) and with a gasrich major galaxy merger (Wang et al. 2012, total mass ratio 


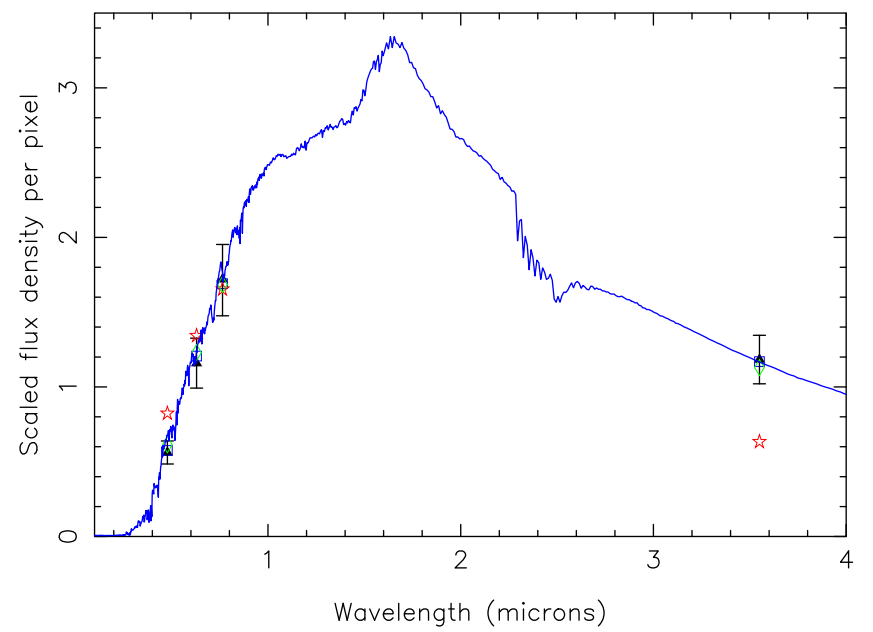

Figure 9. Best fit of an FSPS/Padova model to the stellar stream SED around NGC 5907. The observations are shown with black filled triangles. The error bars reflect the uncertainty due to the background subtraction in the Subaru Suprime-Cam observations and the uncertainty in the background determination in the IRAC $3.6 \mu \mathrm{m}$ mosaic. The best fitting FSPS/Padova model (age $14.96 \mathrm{Gyr}$ and metallicity -0.3 ) is shown with blue open squares and the corresponding model spectrum with a solid blue line. A low metallicity model (age $9.44 \mathrm{Gyr}$ and metallicity -1.98 , to demonstrate that low metallicity models are ruled out) is shown with open red stars, and a model with an age of $10 \mathrm{Gyr}$ and metallicity of -0.3 with open green diamonds. The $y$-axis is in arbitrary surface brightness units.

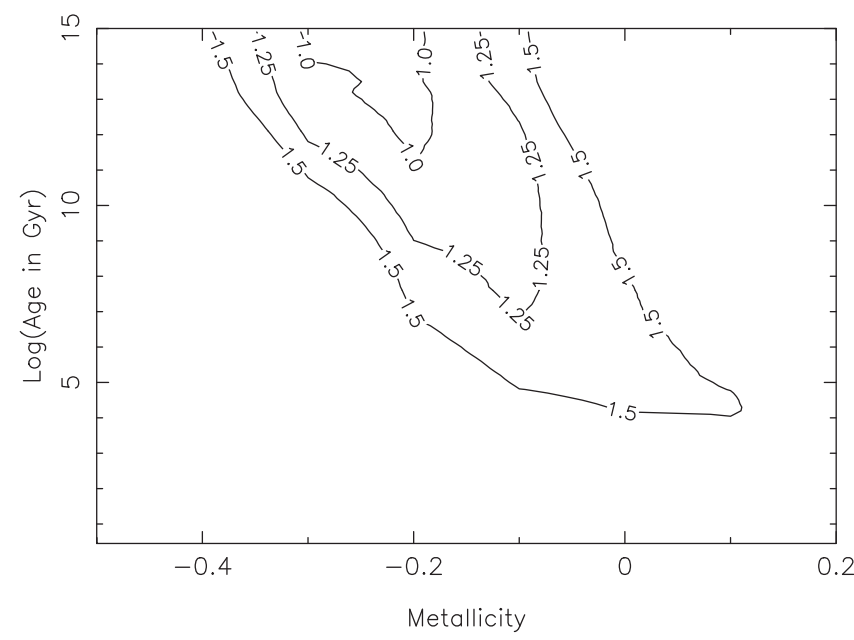

Figure 10. $\chi^{2}$ values of the fits of the FSPS/Padova models to the data, in agemetallicity space. The contours correspond to about $87 \%\left(\chi^{2}=1.25\right)$ and $83 \%$ $\left(\chi^{2}=1.5\right)$ confidence limits for four degrees of freedom.

1:3-1:5). Neither $N$-body model achieved a quantitative fit to the stream, but both were able to qualitatively create features similar to those seen in the observations.

The main objection to the satellite galaxy accretion model is that there is no obvious remnant of the nuclear region of the disrupted satellite (Wang et al. 2012). In NGC 5387 Beaton et al. (2014) found signs of a very blue star-forming cluster right at the intersection of a stellar stream and the host galaxy disk. They speculated that this could be the starbursting nuclear remnant of the mostly disrupted satellite galaxy. However, no such star-forming region has been found near the intersections of the disk of NGC 5907 and the stellar stream, and we do not see any such blue star-forming region along the brightest section of the stellar stream that we have imaged. Nor have we detected any signs of active star formation in the stellar stream.

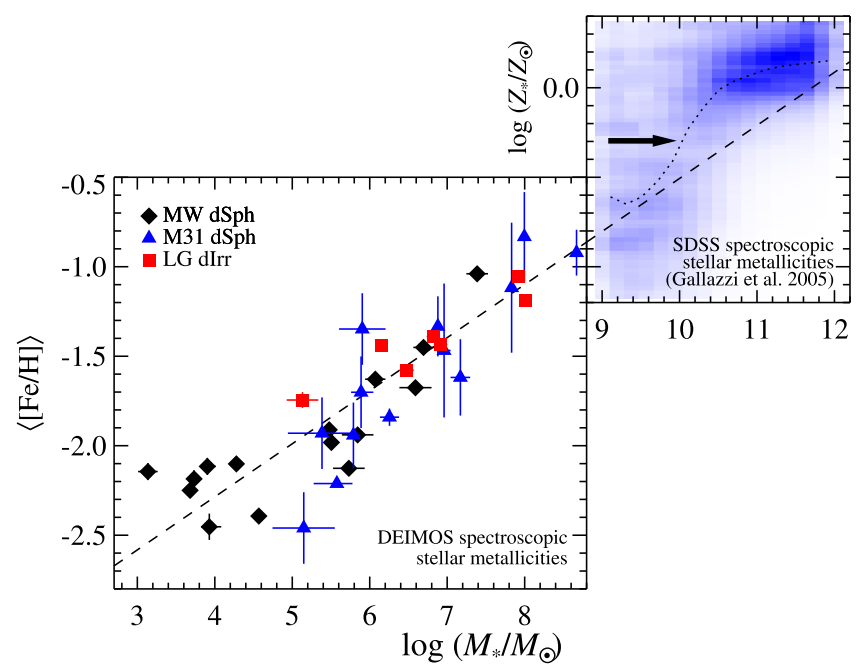

Figure 11. Stellar mass-stellar metallicity relation, reproduced from Figure 9 of "The Universal Stellar Mass-Stellar Metallicity Relation for Dwarf Galaxies" by Kirby et al. (2013). The lower left shows the relation for local group dwarf galaxies and the upper right for more massive galaxies in SDSS from Gallazzi et al. (2005). The dotted line in the diagram for the more massive galaxies is the median of the stellar metallicity distribution as a function of stellar mass. The black arrow was added to point to the metallicity value measured in this work for NGC 5907. See Kirby et al. (2013) for more information.

The main objection to the major galaxy merger model is that NGC 5907 appears to have a thin disk with no obvious bulge (see Athanassoula et al. 2016) and does not look like a severely perturbed major merger remnant (see major merger simulations by, e.g., Barnes 1992). In the gas-rich major merger scenario, the thin disk of NGC 5907 is rebuilt around a small bulge. A similar claim for the creation of the faint stellar stream features around NGC 4013 by a major merger has recently been made by Wang et al. (2015). Another objection to the major merger model could be the lack of any detected neutral atomic hydrogen (H I) gas in the stellar stream (Shang et al. 1998) if it is interpreted as being a long remnant tidal tail.

Our results suggest that the stream in NGC 5907 is relatively metal-rich: the best fitting FSPS model has a metallicity $\log \left[Z / Z_{\odot}\right](\approx[\mathrm{Fe} / \mathrm{H}]$, assuming that the fractional percentage of hydrogen is the same as in the Sun and that the iron abundance and the total metal abundances are roughly the same $)=-0.3$. This should be compared to the reported metallicity in the Sagittarius stream around the Milky Way, from a peak $[\mathrm{Fe} / \mathrm{H}]$ of -0.3 in its core to a median $[\mathrm{Fe} / \mathrm{H}]$ from -0.7 to -1.1 in its leading arm (Chou et al. 2007). The Sagittarius stream is known to be an interacting satellite galaxy, and its core has been identified. While the bound mass of the Sagittarius dwarf (the progenitor of the Sagittarius stream) is currently about $2.5 \times 10^{8} M_{\odot}$, the $N$-body modeling of Law \& Majewski (2010) suggests an original mass of $6.4 \times 10^{8} M_{\odot}$. Meanwhile, in the giant southern stellar stream of M31, the $[\mathrm{Fe} / \mathrm{H}]$ has a strong peak at -0.3 , with a mean of -0.55 and a median of -0.45 (Guhathakurta et al. 2006; Kalirai et al. 2006; Tanaka et al. 2010). The disrupted precursor galaxy for the giant southern stellar stream in M31 could have been a satellite of stellar mass $4 \times 10^{9} M_{\odot}$ (Dekel \& Woo 2003; Tanaka et al. 2010). A comparison to the $g-i$ color of about $0.6-0.8$ for the umbrella in NGC 4651 (Foster et al. 2014) that is supposedly a result from a merger of a metal-poor satellite 
suggests that the merging galaxy in the NGC 5907 system was more metal-rich.

Using the metallicity of the NGC 5907 stellar stream and the metallicity versus stellar mass relationship in Dekel \& Woo (2003) and Kirby et al. (2013, reproduced in Figure 11 of the current paper), the disrupted companion of NGC 5907 would have had a stellar mass of about $1 \times 10^{10} M_{\odot}$, with a large uncertainty that extends down to $\sim 1 \times 10^{9} M_{\odot}$. Note that the mass-metallicity relationship evolves when going to a higher redshift sample. Similarly, Equation (9) in Kirby et al. (2011) predicts $L_{\mathrm{tot}}=4.1 \times 10^{10} L_{\odot}$ for the disrupted companion of NGC 5907, using our derived $[\mathrm{Fe} / \mathrm{H}]$ value of -0.3 . This would make this system qualify as a major merger, because the dynamical disk mass in NGC 5907 has been estimated to be about $1.4 \times 10^{11} M_{\odot}$ (Casertano 1983, adjusted for the adopted distance of $17 \mathrm{Mpc}$ in our study), of which gas may constitute about 10\% (Dumke et al. 1997). Just et al. (2006) modeled the disk of NGC 5907 with a stellar mass of $2 \times 10^{10} M_{\odot}$, making the merger an almost equal disk mass merger. However, using the conversion from IRAC 3.6 and $4.5 \mu \mathrm{m}$ flux densities by Eskew et al. (2012), the stellar mass of NGC 5907 is about $7.8 \times 10^{10} M_{\odot}$. We adopt this as the best estimate to compare with our (partly) IRAC-based metallicity measurement.

We have estimated the mass in the IRAC-imaged part of the stellar stream (Table 1). The value we obtain, $2.1 \times 10^{8} M_{\odot}$, is consistent with the value estimated by Martínez-Delgado et al. (2008), $3.5 \times 10^{8} M_{\odot}$, because Martínez-Delgado et al. (2008) used the luminosity of the whole stream that is seen in Figure 1 to estimate the mass. This mass would imply a satellite accretion event that may also be consistent with our color findings if one allows for systematic issues in the stellar population modeling (see Section 4). On the other hand, the estimated stream mass is only a lower limit to the progenitor mass and thus it cannot accurately constrain the merger scenario, because in a minor merger most of the merged companion mass has presumably become part of the bulge and disk of the primary galaxy, NGC 5907.

\section{CONCLUSIONS}

We have studied the brightest part of the stellar stream around NGC 5907 with both visible-light $(g, i$, and $r$ ) and IRAC near-infrared $3.6 \mu \mathrm{m}$ images. While the stream is very faint, we have managed to generate color index profiles and an SED for the brightest part of the stream. Our results show a slight color change in some of the bands along the stream, most notably in $g-i$ and $r-i$ in which the color becomes bluer after the turning point in the northeast corner of the brightest parts of the stream. However, there may be a remaining background subtraction problem in the $i$ band and our results are essentially consistent with no color gradients along the stream.

We have fitted FSPS stellar population synthesis models with a single-age stellar population. The best fit is achieved for an old and relatively metal-rich stellar population in the stream. Such a stellar population appears to be more consistent with a rather massive minor merger event than with the stripped stars from a dwarf galaxy accretion event.

Future work should include better visible light photometry of the stream, and improving the stellar population synthesis model predictions in the near-infrared. Future kinematical studies of globular clusters associated with this stream could shed light on the implied minor merger scenario (A. B. Alabi et al. 2016, in preparation).

We thank the referee, Rodrigo Ibata, for critical comments that greatly improved the paper. We are indebted to David Shupe for help with fitting the stellar population synthesis models. We thank Charlie Conroy, Ben Johnson and Alexa Villaume for helpful discussions regarding stellar population synthesis codes. We thank Ben Johnson for his help with the Python interface to FSPS. We thank Sean Carey for useful discussions on error estimation. And we thank Evan Kirby and Anna Gallazzi for permission to reproduce a figure from their paper. D.M.D. was supported by Sonderforschungsbereich SFB 881 "The Milky Way System" (subproject A2) of the German Research Foundation (DFG). This research was supported in part by the National Science Foundation under grant Nos. AST-1211995 and AST-1518294. This work is based in part on observations made with the Spitzer Space Telescope, which is operated by the Jet Propulsion Laboratory, California Institute of Technology under a contract with NASA. Support for this work was provided by NASA through an award issued by JPL/Caltech. Based in part on data collected at Subaru Telescope, which is operated by the National Astronomical Observatory of Japan. This research has made use of the NASA/IPAC Extragalactic Database (NED), which is operated by the Jet Propulsion Laboratory, California Institute of Technology, under contract with the National Aeronautics and Space Administration. This research has made use of the NASA/IPAC Infrared Science Archive, which is operated by the Jet Propulsion Laboratory, California Institute of Technology, under contract with the National Aeronautics and Space Administration.

Facilities: Spitzer, Subaru (Suprime-Cam).

Software: MOPEX, SExtractor, Adobe Photoshop.

\section{REFERENCES}

Arendt, R. G., Fixen, D. J., \& Moseley, S. H. 2000, ApJ, 536, 500

Arp, H. 1966, ApJS, 14, 1

Athanassoula, E., Rodionov, S. A., Peschken, N., \& Lambert, J. C. 2016, ApJ, 821, 90

Barnes, J. E. 1992, ApJ, 393, 484

Beaton, R. L., Martínez-Delgado, D., Majewski, S. R., et al. 2014, ApJ, 790, 117

Belokurov, V., Evans, N. W., Irwin, M. J., et al. 2007, ApJ, 658, 337

Bertin, E., \& Arnouts, S. 1996, A\&A, 117, 393

Brown, T. M., Smith, E., Ferguson, H. C., et al. 2006, ApJ, 652, 323

Bullock, J. S., \& Johnston, K. V. 2005, ApJ, 635, 931

Bush, S. J., Wang, Z., Karovska, M., \& Fazio, G. G. 2008, ApJ, 688, 875

Capaccioli, M., \& de Vaucouleurs, G. 1983, ApJS, 52, 465

Capak, P., Aussel, H., Ajiki, M., et al. 2007, ApJS, 172, 99

Casertano, S. 1983, MNRAS, 203, 735

Chou, M.-Y, Majewski, S. R., Cunha, K., et al. 2007, ApJ, 670, 346

Conroy, C., \& Gunn, J. E. 2010, ApJ, 712, 833

Conroy, C., Gunn, J. E., \& White, M. 2009, ApJ, 699, 486

Cordier, D., Pietrinferni, A., Cassisi, S., \& Salaris, M. 2007, AJ, 133, 468

Deason, A. J., Mao, J.-J., \& Wechsler, R. 2016, ApJ, 821, 5

de Boer, T. J. L., Belokurov, V., \& Koposov, S. 2015, MNRAS, 451, 3489 Dekel, A., \& Woo, J. 2003, MNRAS, 344, 1131

Duc, P.-A., Cuillandre, J.-C., Karabal, E., et al. 2015, MNRAS, 446, 120

Dumke, M., Braine, J., Krause, M., et al. 1997, A\&A, 325, 124

Eskew, M., Zaritsky, D., \& Meidt, S. 2012, AJ, 143, 139

Fazio, G. G., Hora, J. L., Allen, L. E., et al. 2004, ApJS, 154, 10

Fixen, D. J., Moseley, S. H., \& Arendt, R. G. 2000, ApJS, 128, 651

Foreman-Mackey, D., Johnson, B., \& Sick, J. 2014, python-fsps: Python bindings to FSPS (v0.1.1) Zenodo

Foster, C., Lux, H., Romanowsky, A. J., et al. 2014, MNRAS, 442, 3544 
Gallazzi, A., Charlot, S., Brinchmann, J., White, S. D. M., \& Tremonti, C. A. 2005, MNRAS, 362, 41

Girardi, L., Bressan, A., Bertelli, G., \& Chiosi, C. 2000, A\&AS, 141, 371

Grillmair, C. 2006a, ApJL, 645, L37

Grillmair, C. 2006b, ApJL, 651, L29

Grillmair, C. 2009, ApJ, 693, 1118

Grumm, D., Casertano, S., Dickinson, M., \& Holfeltz, S. 2005, in ASP Conf. Series 347, Astronomical Data Analysis Software and Systems XIV, ed. P. Shopbell, M. Britton, \& R. Ebert, (San Francisco, CA: ASP), 454

Guhathakurta, P., Rich, R. M., Reitzel, D. B., et al. 2006, AJ, 131, 2497

Hibbard, J. E., \& van Gorkon, J. H. 1996, AJ, 111, 655

Ibata, R., Irwin, M., Lewis, G., Ferguson, A. M. N., \& Tanvir, N. 2001a, Natur, 412, 49

Ibata, R., Lewis, G. F., Irwin, M., Totten, E., \& Quinn, T. 2001b, ApJ, 551, 294 Jennings, Z. G., Romanowsky, A. J., Brodie, J. P., et al. 2015, ApJL, 812, L10

Jester, S., Schneider, D. B., Richards, G. T., et al. 2005, AJ, 130, 873

Just, A., Möllenhoff, C., \& Borch, A. 2006, A\&A, 459, 703

Kalirai, J. S., Guhathakurta, P., Gilbert, K. M., et al. 2006, ApJ, 641, 268

King, I. R. 1971, PASP, 83, 199

Kirby, E. N., Cohen, J. G., Guhathakurta, P., et al. 2013, ApJ, 779, 102

Kirby, E. N., Lanfranchi, G. A., Simon, J. D., Cohen, J. G., \& Guhathakurta, P. 2011, ApJ, 727, 78

Kroupa, P. 2001, MNRAS, 322, 231

Law, D. R., \& Majewski, S. R. 2010, ApJ, 714, 229

Majewski, S. R., Skrutskie, M. F., Weinberg, M. D., \& Ostheimer, J. C. 2003, ApJ, 599, 1082

Marigo, P., \& Girardi, L. 2007, A\&A, 469, 239

Marigo, P., Girardi, L., Bressan, A., et al. 2008, A\&A, 482, 883

Martínez-Delgado, D., D’Onghia, E., Chonis, T. S., et al. 2015, AJ, 150, 116

Martínez-Delgado, D., GaBany, R. J., Crawford, K., et al. 2010, AJ, 140, 962
Martínez-Delgado, D., Peñarrubia, J., GaBany, R. J., et al. 2008, ApJ, 689, 184 Martínez-Delgado, D., Pohlen, M., GaBany, R. J., et al. 2009, ApJ, 692, 955 Michard, R. 2002, A\&A, 384, 763

Miyazaki, S., Komiyama, Y., Sekiguchi, M., et al. 2002, PASJ, 54, 833

Mori, M., \& Rich, R. M. 2008, ApJL, 674, L77

NASA/IPAC Infrared Science Archive Spitzer Survey of Stellar Structure in Galaxies (Pasadena, CA: IRSA) S4G, http://irsa.ipac.caltech.edu/data/ SPITZER/S4G/

Newberg, H. J., Yanny, B., Rockosi, C., et al. 2002, ApJ, 569, 245

Pietrinferni, A., Cassisi, S., Salaris, M., \& Castelli, F. 2004, ApJ, 612, 168

Planck Collaboration, Ade, P. A. R., Aghanim, M., et al. 2016, A\&A, in press (arXiv:1502.01589)

Purcell, C. W., Bullock, J. S., \& Kazantzidis, S. 2010, MNRAS, 404, 1711

Rodriguez-Gomez, V., Genel., S., Vogelsberger, M., et al. 2015, MNRAS, 449, 49

Sandin, C. 2014, A\&A, 567, A97

Schweizer, F. 1982, ApJ, 252, 455

Shang, Z., Zheng, Z., Brinks, E., et al. 1998, ApJL, 504, L23

Stewart, K. R., Bullock, J. S., Barton, E. J., \& Wechsler, R. H. 2009, ApJ, 702, 1005

Tanaka, M., Chiba, M., Komiyama, Y., et al. 2010, ApJ, 708, 1168

Tully, R. B., Courtois, H. M., Dolphin, A. E., et al. 2013, AJ, 146, 86

Villaume, A., Conroy, C., \& Johnson, B. D. 2015, ApJ, 806, 82

Wang, J., Hammer, F., Athanassoula, E., et al. 2012, A\&A, 538, A121

Wang, J., Hammer, F., Puech, M., Yang, Y., \& Flores, H. 2015, MNRAS, 452, 3551

Werner, M. W., Roellig, T. L., Low, F. J., et al. 2004, ApJS, 154, 1

$\mathrm{Xu}, \mathrm{C}$. K., Zhao, Y., Scoville, N., et al. 2012, ApJ, 747, 85

Zaritsky, D., \& Rix, H.-W. 1997, ApJ, 477, 118

Zheng, Z., Shang, Z., Su, H., et al. 1999, AJ, 117, 2757 